\title{
Intraoperative frozen cytology of intraosseous cystic meningioma in the sphenoid bone
}

\author{
Na Rae Kim ${ }^{1}$, Gie-Taek Yie ${ }^{2}$ \\ Departments of ${ }^{1}$ Pathology and ${ }^{2}$ Neurosurgery, Gil Medical Center, Gachon University College of Medicine, Incheon, Korea
}

\begin{abstract}
Meningiomas in bone are rarely subjected to fine-needle aspiration diagnosis, and those arising in the skull bone with a cystic presentation are rare. A 24-year-old woman presented with subdural hemorrhage, and subsequent radiology depicted an osteolytic mass-like lesion in the sphenoid bone. Intraoperatively, a solid and cystic hemorrhagic lesion mimicking an aneurysmal bone cyst was observed in the sphenoid bone with dural tearing. Frozen cytology showed singly scattered or epithelioid clusters of round to elongated cells intermixed with many neutrophils. Tumor cells had bland-looking round nuclei with rare prominent nucleoli and nuclear inclusions and eosinophilic granular to globoid cytoplasm in capillary-rich fragments. Histology revealed intraosseous meningothelial and microcystic meningioma (World Health Organization grade 1) in right lesser wing of the sphenoid bone. Considering its unusual location and cytologic findings, differential diagnoses included chordoma, chondroma, chondrosarcoma, and aneurysmal bone cyst. The present case posed a diagnostic challenge due to possible confusion with these entities.
\end{abstract}

Key Words: Meningioma; Skull; Sphenoid bone; Osteolysis; Bone cysts, aneurysmal

Received: April 21, 2020 Accepted: May 21, 2020

Corresponding Author: Na Rae Kim, MD, Department of Pathology, Gil Medical Center, Gachon University College of Medicine, 21 Namdong-daero 774 beon-gil, Namdong-gu, Incheon 21565, Korea

Tel: +82-32-460-3073, Fax: +82-32-460-2394, E-mail: clara_nrk@gilhospital.com

Meningiomas are slow-growing mesenchymal neoplasms of the central nervous system and usually arise from the meningothelial cells of the arachnoid layer of meninges. Primary extradural meningioma constitutes $1 \%$ to $2 \%$ of all meningiomas [1]. Intraosseous meningioma is a rare form of bone tumor [2]. Cystic meningioma is uncommon [3], and intraosseous meningioma affecting the skull is also rare. Since the first report of intraosseous meningioma issued by Winkler in 1904, slightly more than 100 cases have been reported [4], and few of these cases concerned cystic osteolytic intraosseous meningioma in the skull [5]. Main differential diagnosis was aneurysmal bone cyst (ABC) that occupies only 2\%-6\% of the skull base [6].

We report a rare case of osteolytic intraosseous meningioma of the sphenoid bone in a 24-year-old woman, and describe intraoperative imprint smears produced using intraosseous samples and the clinicoradiologic resemblance of this disease entity with other intracranial lesions.

\section{CASE REPORT}

A previously healthy 24-year-old woman presented with a headache of two days duration. She was referred to the emergency room of our hospital because she had a history of slip-down in a bathroom two days previously. Precontrast brain computed tomography (CT) revealed a small amount of extra-axial hemorrhage at the right frontotemporal region (Fig. 1A). Skull magnetic resonance images showed a slightly rim-enhancing lesion invading right sphenoid bone. Differential diagnoses included vascular pathologies such as hemangioma and other tumorous conditions. The skull tumor was removed using a navigation system and cranioplasty of the right frontotemporal area was performed using bone cement. Inferolateral temporal dura over the lesion had been focally disrupted, but no gross leakage was observed. After exposing the right temporal fascia and muscle, the lateral wall of the right orbit, zygoma, and a thinned portion of the bone at the pterygoid process were drilled. Removal of the hematoma exposed a multiseptated cystic cavity (Fig. 1B). 

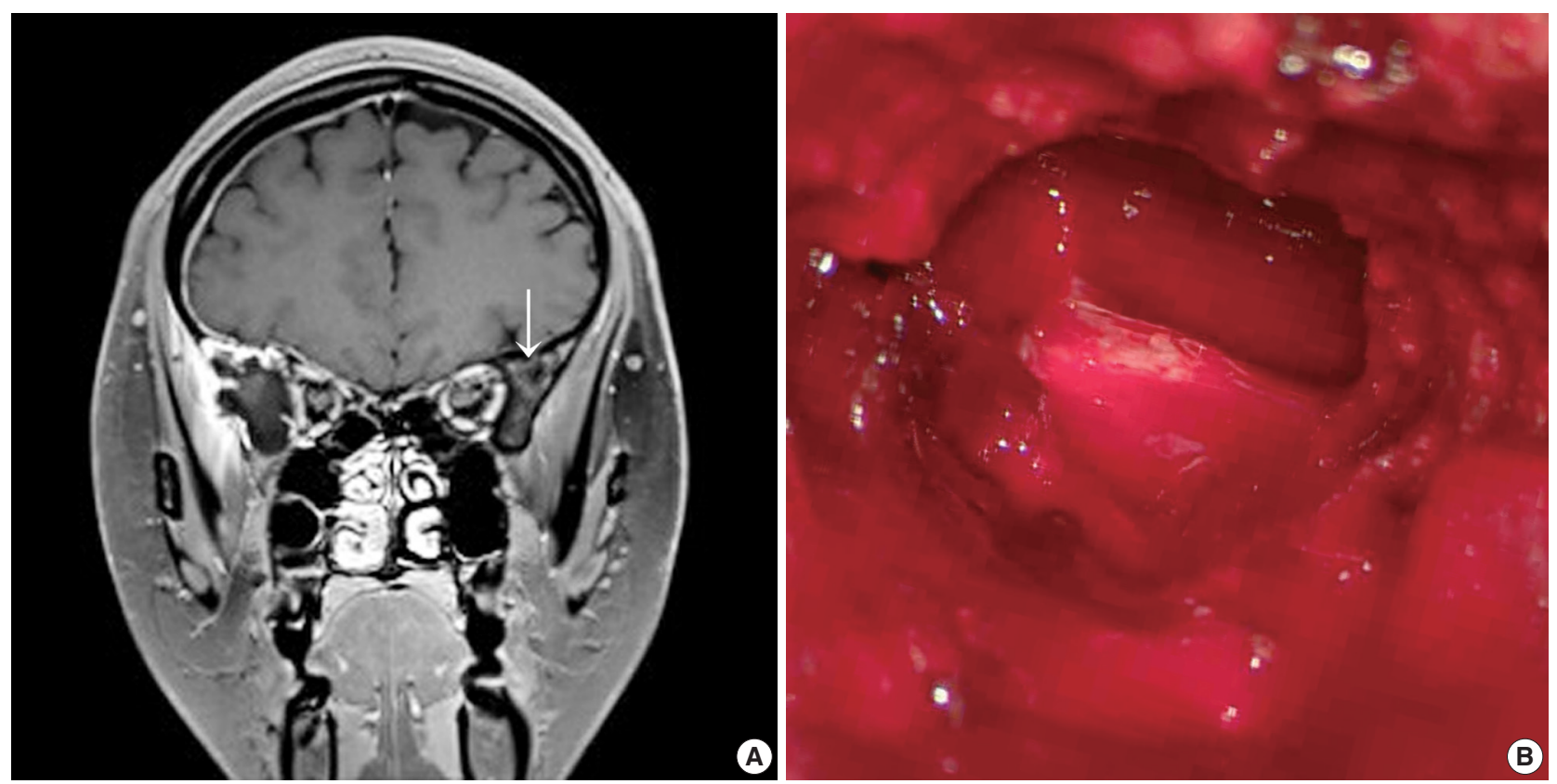

Fig. 1. (A) Brain magnetic resonance image shows a slightly enhanced lesion (arrow) at the right sphenoid bone. (B) Intraoperative gross examination of the lesion shows cystic spaces filled with blood and solid texture.

Intraoperative frozen biopsy was performed on the cystic portion of right sphenoid bone, and subsequent intraoperative squash cytology revealed moderate cellular smears composed of sheets or clusters of epithelioid cells in an inflammatory cellrich background (Fig. 2A-D). Histologically, singly scattered or clusters of round-to-elongated tumor cells were found in sclerotic bone (Fig. 2E). Tumor cells with indistinct cell borders were observed in clusters forming meningothelial whorls and had centrally-placed small round and bland nuclei with or without nucleoli. Prominent hemorrhage was present. Individual tumor cells showed abundant eosinophilic cytoplasm with an occasional vacuolated appearance and cytoplasmic clearing resembling oligodendroglioma (Fig. 2F). Neutrophils were abundant (Fig. 2G, left). There was marked perivascular hyalinization and stromal sclerosis (Fig. 2G, right). Mitotic counts were up to 1 per 10 high power fields, necrosis was absent, and occasional atypia was observed. Tumor cells invaded the thick dura mater. They were positive for CD56 (1:100, CD564, Novocastra, Newcastle upon Tyne, UK), CD99 (prediluted, 12E7, Dako, Glostrup, Denmark), INI-1 (1:200, 25/BAF47, BD Bioscience, San Diego, CA, USA), vimentin (1:100, V9, Dako) (Fig. 2H, left), and progesterone receptor (1:200, 16, Novocastra). Tumor cells also displayed focally membranous positivity for epithelial membrane antigen (1:100, E29, Dako) (Fig. 2H, right). However, they were negative for glial fibrillary acidic protein (prediluted, Dako), S-100 protein (polyclonal, 1:600, Dako), smooth muscle actin
(1:100, 1A4, Dako), synaptophysin (prediluted, DAK-SYNAP, Dako), chromogranin (1:100, DAK-A3, Dako), CD34 (1:100, QBEnd10, Dako), L1 cell adhesion molecule (1:200, polyclonal, Abcam, Cambridge, UK), OLIG2 (EP112; 1:500, Cell Marque, Rocklin, CA, USA), and STAT6 (S-20, sc-621, 1:150, Santa Cruz Biotechnology, Santa Cruz, CA, USA). The Ki-67 (MIB-1, 1:100, Dako) proliferation index was 3\%. Intervening sclerosis stained with periodic acid-Schiff and Masson-trichrome.

Based on the above histology, notably, the clear cell morphology containing one mitosis, meningothelial whorls, and the lack of hypercellularity, macronuclei, small cell formation, or brain invasion, the tumor was determined to be an intraosseous meningioma of the microcystic and meningotheliomatous type (World Health Organization grade I). We diagnosed this case as intraosseous meningioma with dural involvement because the tumor was largely located within the sphenoid bone.

At 5 months of follow-up, the patient was healthy, asymptomatic, and scheduled for annual postoperative follow-ups.

\section{DISCUSSION}

Intraosseous occurrence of meningioma, especially in the skull, is uncommon [7]. Plausibly, this rare primary intraosseous meningioma may arise due to aberrant differentiation or localization of pluripotent embryonal precursor cells of the bone, or from misplaced or trapped arachnoid cells along the suture line dur- 
ing molding of the head at birth and after differentiation into meningocytes or arachnoid cap cells [1]. Alternatively, it may arise from arachnoidal cells of blood vessels or nerves traversing the skull [8].

Intraosseous meningioma of the skull can be confused with primary calvarial bone tumors. On CT images, intraosseous me-
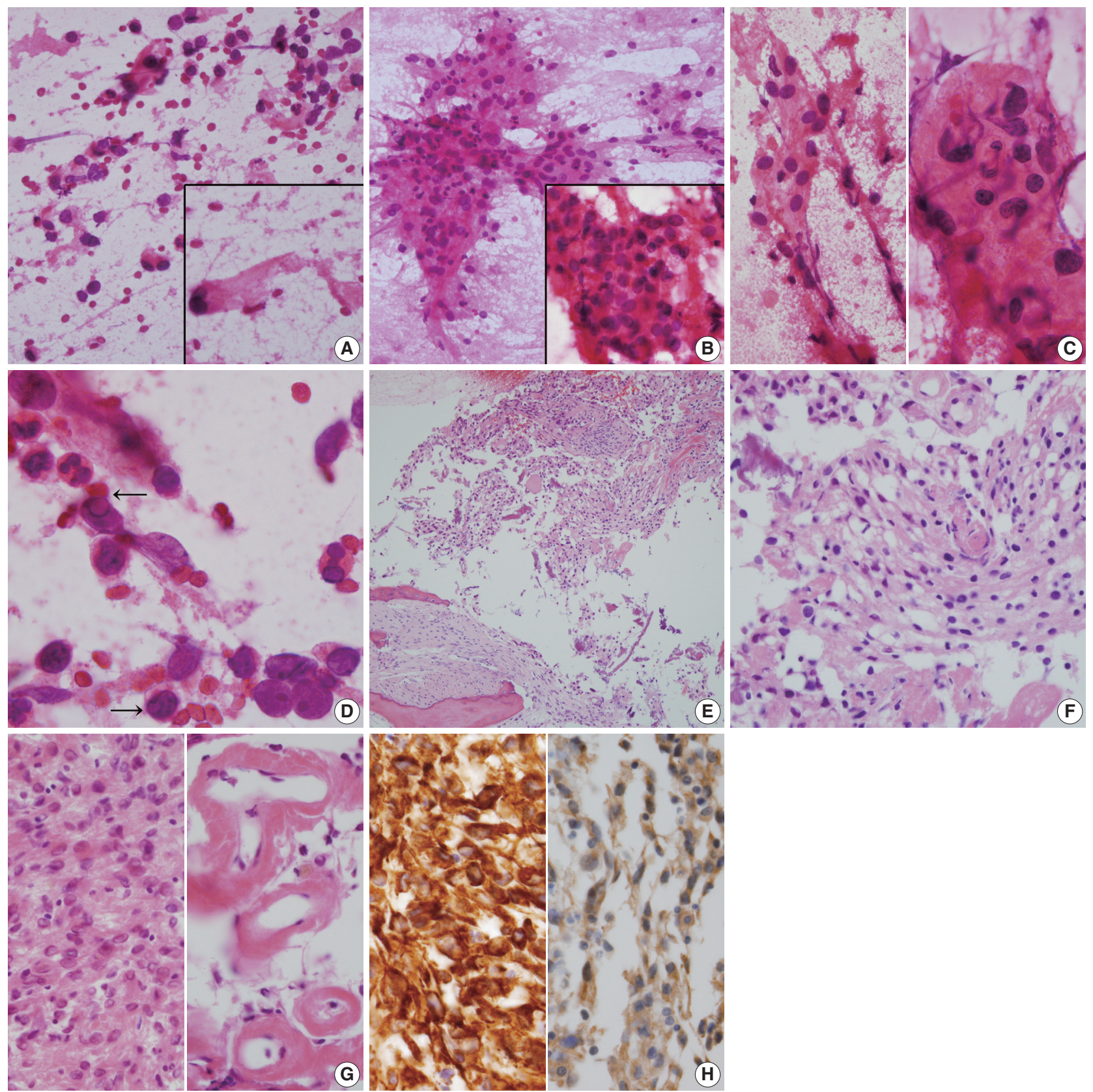

Fig. 2. (A-D) Frozen cytology. (A) The cystic portion of the right sphenoid bone shows singly scattered eosinophilic round to elongated cells with occasional nuclear hyperchromasia. Inset shows thick refractile collagenous cytoplasm with nuclear hyperchromasia. (B) Moderately cellular smears shows sheets of epithelioid cells in a bloody and inflammatory cell-rich background. Inset shows intermixed inflammatory cells in meningothelial whorls. (C) Cellular sheets of round-shaped epithelioid cells with moderate amounts of eosinophilic granular cytoplasm and euchromatic round nuclei, just apposed to the capillary (left). High magnification shows indistinct cell borders (right). (D) High power view shows occasional nuclear inclusions (arrows). (E-H) Histology. (E) Low power view shows sheets of spindle to round-shaped tumor cells, filling the bone. Note the lamellated bone and osteoblastic rimming. (F) Nests of meningothelial cells form microcystic cobweb-like arrangement. Note the microcystic and bubbly cytoplasm. (G) Sheets of spindle-shaped tumor cells are intermixed with inflammatory cells (left). Note the perivascular sclerosis (right). $(\mathrm{H})$ The tumor cells are immunoreactive for vimentin (left) and epithelial membrane antigen (right). 
ningiomas appear as osteoblastic, osteolytic, or mixed patterns $[3,9]$. Skull lesions showing osteolytic lesions, as in our case, include epidermoid tumor, multiple myeloma, Langerhans cell histiocytosis, fibrous dysplasia, giant-cell tumor, Paget's disease, solitary fibrous tumor/hemangiopericytoma (SFT/HPC), hemangioma, osteosarcoma, and $\mathrm{ABC}[10]$. In the present case, the lesion was located in the sphenoid bone and its preoperative radiologic and gross differential diagnoses included $A B C$, chordoma, chondrosarcoma, and vascular neoplasm [11]. ABC presenting as a locally destructive osteolytic lesion frequently involves the long bones of knee joints and vertebrae and is usually encountered in the first or second decades of life. When ABC occurs in the skull, it is usually located in the skull vault and only rarely in the skull base [11]. The preoperative diagnosis of an intraosseous lytic lesion like meningioma is challenging. The presence of an osteolytic extracranial soft tissue lesion usually raises the suspicion of malignancy [9]. Radiologic differential diagnosis of osteolytic intraosseous meningiomas of the skull should exclude other lytic lesions such as lytic metastasis, multiple myeloma, eosinophilic granuloma, fibrous dysplasia, Langerhans cell histiocytosis, giant-cell tumor, SFT/HPC, and hemangioma. Radiologically, intraosseous meningiomas present osteoblastic findings in about two-thirds of cases, an osteolytic pattern, in around a third, and a mixed osteoblastic-lytic lesion in only $6 \%$ of cases [2,9].

Evaluations of skull lesions by fine-needle aspiration are controversial. Cytologic findings of meningioma are diverse and heterogeneous [12], and notable features for differential diagnosis are as follows; cohesive syncytial meningothelial whorls, individual tumor cells showing nuclear inclusions or grooves and wispy collagenous, rather than fibrillary, cytoplasm with occasional short broad cell processes [13]. Tumor cells with indistinct cell borders and subsequent syncytial whorls are the most common. In meningothelial and microcystic type meningiomas, as in the present case, background collagenous sclerosis is observed in tumor cells with vacuolated cytoplasm mixed with scattered inflammatory cells [13]. Although the biologic roles of circulating neutrophils and intratumoral neutrophils in meningiomas remain unclear, the presence of many inflammatory cells including lymphocytes, plasma cells, natural killer cells, and macrophages is common [14]. A high concordance between frozen and permanent diagnoses (up to 99\%) has been reported for meningeal tumors [12]. However, frozen cytologic diagnosis in the described case was difficult due to the presence of massive numbers of inflammatory cells, marked hemorrhage, and an unusual location. Scattered stellate, vacuolated cells, and foam cells may be found in regions of microcystic meningiomas [13]. Singly-scattered tumor cells with intracytoplasmic vacuoles in foci of microcystic meningioma resembling atypical chondrocytes of chondrosarcoma and lipoblasts of liposarcoma were occasionally found [15]. Cytologic findings of chondrosarcoma are moderately cellular with clusters of polygonal cells showing occasional binucleation and well-defined cytoplasm with rounded nuclei showing moderate nuclear pleomorphism embedded in a chondromyxoid background, suggestive of chondrosarcoma [15]. Cytologic findings of chordoma are characterized by mucoid and gelatinous fibromyxoid cellular smears and cords or clusters of round epithelioid cells with small round nuclei and scant basophilic cytoplasm and occasional large physaliphorous cells with central small round nuclei and bubbly cytoplasm [16]. Well differentiated chondrosarcoma arising in the sphenoid bone is another entity necessitating differential diagnosis; the findings of bland nuclei and refractile cytoplasmic processes and a lack of chondromyxoid matrix are useful diagnostic features of meningioma [12].

The most important macroscopic frozen differential diagnosis in the described case was $\mathrm{ABC}$. Cytologic findings of $\mathrm{ABC}$ are dominated by blood; dispersed fibroblast-like histiocytes with occasional hemosiderin and multinucleated osteoclast-type giant cells containing fragments of collagen [17]. Osteoid and chondroid matrix material as well as woven bone may be present within aspirated or touch smears. Small fragments of bone may also be found. In hemorrhagic cases, as in our case, markedly vascular hemorrhagic SFT/HPC should be distinguished from meningioma. Cytologic findings of SFT/HPC are nonspecific and "patternless", i.e., heterogeneous, although naked stripped nuclei and scattered ropy collagen fragments are observed in an occasional pseudoalveolar pattern in a hemorrhagic bloody background [18]. The histologic diagnosis of intraosseous meningioma is not as difficult as that based on frozen cytology. One of the important histologic differential diagnoses of meningioma is SFT/HPC. Immunopositivity for both CD34 and STAT6 in SFT/HPC differentiate it from meningioma [19]. At a glance, clear cells mimicking oligodendroglial cells suggest oligodendroglioma [20]. However, oligodendroglioma and meningioma can be differentiated based on the presence of whorls and immunonegativity for OLIG2 in the meningioma. Histologically, ABC features fibroblastic proliferation and scattered giant cells with osteoid and blood-filled spaces lined by giant cells and hemosiderin laden macrophages [17].

Awareness of intraosseous cystic meningioma involving the skull may facilitate the intraoperative diagnosis of this rare condition. The present case cautions that the possibility of intraos- 
seous meningioma should be kept in mind when is it is encountered in an unusual location.

\section{Ethics Statement}

An approval was obtained from our Institutional Review Board (No. GDIRB2020-122) for publication of this case report with a waiver of informed consent.

\section{ORCID}

Na Rae Kim Gie-Taek Yie

https://orcid.org/0000-0003-2793-6856 https://orcid.org/0000-0002-8706-7253

\section{Author Contributions}

Conceptualization: NRK. Data curation: NRK. Investigation: GTY. Resources: GTY. Supervision: NRK. Validation: GTY. Visualization: GTY. Writing—original draft: NRK. Writing—review \& editing: NRK. Approval of final manuscript: all authors.

\section{Conflicts of Interest}

The authors declare that they have no potential conflicts of interest.

\section{Funding Statement}

No funding to declare.

\section{References}

1. Lang FF, Macdonald OK, Fuller GN, DeMonte F. Primary extradural meningiomas: a report on nine cases and review of the literature from the era of computerized tomography scanning. J Neurosurg 2000; 93: 940-50.

2. Agrawal V, Ludwig N, Agrawal A, Bulsara KR. Intraosseous intracranial meningioma. AJNR Am J Neuroradiol 2007; 28: 314-5.

3. Rosahl SK, Mirzayan MJ, Samii M. Osteolytic intra-osseous meningiomas: illustrated review. Acta Neurochir (Wien) 2004; 146 1245-9.

4. Butscheidt S, Ernst M, Rolvien T, et al. Primary intraosseous meningioma: clinical, histological, and differential diagnostic aspects. J Neurosurg 2019 Jun 21 [Epub]. https://doi.org/10.3171/2019. 3.JNS182968.

5. Caruso R, Fini G, Pesce A, et al. A primary intraosseous cystic meningioma: case report. Int J Surg Case Rep 2017; 37: 189-92.

6. Aghaghazvini L, Sedighi N, Karami P, Yeganeh O. Skull base aneurysmal bone cyst presented with foramen jugular syndrome and multi-osseous involvement. Iran J Radiol 2012; 9: 157-60.

7. Ilica AT, Mossa-Basha M, Zan E, et al. Cranial intraosseous meningioma: spectrum of neuroimaging findings with respect to histopathological grades in 65 patients. Clin Imaging 2014; 38: 599-604.

8. Turner OA, Laird AT. Meningioma with traumatic etiology: report of a case. J Neurosurg 1966; 24: 96-8.

9. Tokgoz N, Oner YA, Kaymaz M, Ucar M, Yilmaz G, Tali TE. Primary intraosseous meningioma: CT and MRI appearance. AJNR Am J Neuroradiol 2005; 26: 2053-6.

10. Kee TP, Liauw L, Sathiyamoorthy S, Lee HY, Tan GSL, Yu WY Large solitary lytic skull vault lesions in adults: radiological review with pathological correlation. Clin Imaging 2020; 59: 129-43.

11. Ustabasioglu FE, Samanci C, Asik M, et al. Aneurysmal bone cyst of sphenoid bone and clivus misdiagnosed as chordoma: a case report. Brain Tumor Res Treat 2015; 3: 115-7.

12. Kang M, Chung DH, Kim NR, et al. Intraoperative frozen cytology of central nervous system neoplasms: an ancillary tool for frozen diagnosis. J Pathol Transl Med 2019; 53: 104-11.

13. Seok JY, Kim NR, Cho HY, Chung DH, Yee GT, Kim EY. Crush cytology of microcystic meningioma with extensive sclerosis. Korean J Pathol 2014; 48: 77-80.

14. Pinton L, Solito S, Masetto E, et al. Immunosuppressive activity of tumor-infiltrating myeloid cells in patients with meningioma. Oncoimmunology 2018; 7: e1440931.

15. Lee S, Kim NR, Chung DH, Yee GT, Cho HY. Squash cytology of a dural-based high-grade chondrosarcoma may mimic that of glioblastoma in the central nervous system. Acta Cytol 2015; 59: 219-24.

16. Nijhawan VS, Rajwanshi A, Das A, Jayaram N, Gupta SK. Fineneedle aspiration cytology of sacrococcygeal chordoma. Diagn Cytopathol 1989; 5: 404-7.

17. Creager AJ, Madden CR, Bergman S, Geisinger KR. Aneurysmal bone cyst: fine-needle aspiration findings in 23 patients with clinical and radiologic correlation. Am J Clin Pathol 2007; 128: 740-5.

18. Kang M, Kim NR, Chung DH, Yie GT. Frozen cytology of meningeal malignant solitary fibrous tumor/hemangiopericytoma. J Pathol Transl Med 2019; 53: 192-7.

19. Fritchie K, Jensch K, Moskalev EA, et al. The impact of histopathology and NAB2-STAT6 fusion subtype in classification and grading of meningeal solitary fibrous tumor/hemangiopericytoma. Acta Neuropathol 2019; 137: 307-19.

20. Ligon KL, Alberta JA, Kho AT, et al. The oligodendroglial lineage marker OLIG2 is universally expressed in diffuse gliomas. J Neuropathol Exp Neurol 2004; 63: 499-509. 